\title{
SAPONINAS ANTIFÚNGICAS DE Swartzia langsdorffii
}

\author{
Sara Regina de Marqui, Renata Brionízio Lemos, Luciana Ávila Santos, Ian Castro-Gamboa, Alberto José Cavalheiro, \\ Vanderlan da Silva Bolzani e Dulce Helena Siqueira Silva* \\ Instituto de Química, Universidade Estadual Paulista, CP 355, 14801-970 Araraquara - SP, Brasil \\ Liliana Scorzoni, Ana Maria Fusco-Almeida e Maria José Soares Mendes-Giannini \\ Faculdade de Ciências Farmacêuticas, Universidade Estadual Paulista, 14802-901 Araraquara - SP, Brasil \\ Maria Claudia Marx Young e Luce Maria Brandão Torres \\ Seção de Fisiologia e Bioquímica de Plantas, Instituto de Botânica, 01061-970 São Paulo - SP, Brasil
}

Recebido em 8/2/08; aceito em 2/4/08; publicado na web em 29/4/08

\begin{abstract}
ANTIFUNGAL SAPONINS FROM Swartzia langsdorffii. Chromatographic fractionation of the EtOH extract from the leaves of Swartzia langsdorffii afforded the pentacyclic triterpenes oleanolic acid and lupeol, and two saponins: oleanolic acid 3-sophoroside and the new ester 3-O- $\beta$-D-(6'-methyl)-glucopyranosyl-28- $O-\beta-\mathrm{D}$-glucopyranosyl-oleanate. Their structures were elucidated from spectral data, including 2D NMR and HRESIMS experiments. Antifungal activity of all isolated compounds was evaluated, using phytopathogens Cladosporium cladosporioides and C. sphaerospermum, and human pathogens Candida albicans, C. krusei, C. parapsilosis and Cryptococcus neoformans.
\end{abstract}

Keywords: Swartzia langsdorffii; saponins; antifungal activity.

\section{INTRODUÇÃO}

Espécies da família Fabaceae são amplamente distribuídas em regiões tropicais e subtropicais e divididas em três subfamílias: Caesalpinoideae, Mimosoideae e Papilionoideae. ${ }^{1} \mathrm{O}$ gênero Swartzia pertence à subfamília Caesalpinoideae e inclui 150 espécies, distribuídas pelas Américas do Sul e Central e África. Na América do Sul, o centro de maior diversidade desse gênero está na região amazônica. No Brasil, além da região amazônica, existe uma considerável representatividade de espécies na região sudeste. ${ }^{1,2}$

Swartzia langsdorffii pertence à subfamília Caesalpinoideae, e é popularmente conhecida como banana-de-papagaio, jacarandá-banana e jacarandá-de-sangue. Na região sudeste, é encontrada nos estados do Rio de Janeiro e de São Paulo nas formações florestais do complexo atlântico, principalmente na Serra do Mar. ${ }^{3}$ A árvore é ornamental, principalmente quando em flor, e seus frutos são muito procurados por várias espécies da fauna, que se alimentam do arilo suculento que envolve parcialmente a semente. Por essa razão, essa árvore é interessante para plantio em áreas degradadas para preservação permanente. ${ }^{3} \mathrm{O}$ gênero Swartzia é caracterizado pela presença de isoflavonóides, saponinas triterpênicas e diterpenos aromáti$\cos ^{2,4-7}$ Espécies deste gênero apresentam constituintes químicos com atividade antimicrobiana, ${ }^{4}$ antifúngica, ${ }^{5}$ moluscicida ${ }^{6}$ e citotóxica, ${ }^{2,7}$ o que mostra a importância da investigação adicional sobre aspectos fitoquímicos e/ou farmacológicos destas espécies.

Em nosso programa de bioprospecção, o extrato de Swartzia langsdorffii destacou-se pela marcante atividade antifúngica frente a fitopatógenos e patógenos humanos. Os fungos são amplamente associados a doenças oportunistas e tem-se observado aumento no número de pacientes com alterações no estado imunológico associado ao vírus da imunodeficiência adquirida (HIV), à quimioterapia do câncer e transplante de órgãos e de sangue. Coincidindo com esse aumento de pacientes imunocomprometidos tem ocorrido um aumento da incidência de micoses sistêmicas humanas. ${ }^{8}$ Cryptococcus neoformans,

*e-mail: dhsilva@iq.unesp.br destacado como uma das principais infecções em pacientes aidéticos, e Candida sp, descrita em 20 a $40 \%$ de pacientes com câncer e em aproximadamente $25 \%$ dos pacientes que recebem transplantes de medula óssea, ${ }^{9}$ são alvos de enorme importância. Apesar da existência de potentes agentes antifúngicos como a anfotericina $\mathrm{B}$, o aparecimento de resistência ou multi-resistência de fungos impõe a necessidade de pesquisas permanentes e o desenvolvimento de novas drogas. ${ }^{810}$ Visto que as plantas produzem uma variedade de compostos com propriedades antifúngicas, é esperado que programas de bioprospecção sistemática informem fontes de substâncias candidatas ao desenvolvimento de novas drogas com estas propriedades. ${ }^{11}$ Estudos de bioprospecção preliminares com plantas da Mata Atlântica levaram à seleção de extratos bioativos, dentre os quais, destacou-se o extrato de Swartzia langsdorffii, coletada na Reserva Juréia-Itatins, em Iguape - SP, que apresentou marcada atividade antifúngica e foi selecionado para estudo fitoquímico visando o isolamento e identificação de seus constituintes químicos bioativos.

\section{PARTE EXPERIMENTAL}

\section{Materiais e equipamentos}

A rotação óptica foi medida em polarímetro Perkin-Elmer modelo 341 usando lâmpada de sódio $(589 \mathrm{~nm})$ a $20{ }^{\circ} \mathrm{C}$. Espectros de massas foram medidos em espectrômetro UltrO-TOFq (Bruker-Daltonics).

As análises foram realizadas em modo eletrospray (negativo, $100 \% \mathrm{MeOH}$, injeção da amostra através de seringa de infusão Harvard Apparatus Pump11, com fluxo de $10 \mu \mathrm{L} / \mathrm{min}$ ). Os experimentos de RMN de ${ }^{1} \mathrm{H} \mathrm{e}{ }^{13} \mathrm{C}$ em uma e duas dimensões foram feitos em instrumento Varian Inova 500 operando a $500 \mathrm{MHz}$ para hidrogênio e $125 \mathrm{MHz}$ para carbono usando seqüências de pulso padrão (Varian). DMSO-d ${ }_{6}$ foi usado como solvente e TMS, como padrão interno. A cromatografia planar (CCD) foi realizada em placas de alumínio cobertas de sílica-gel (sílica $\mathrm{F}_{254}, 0.25 \mathrm{~mm}$, Merck, Darmstadt, Germany) com detecção por luz UV (254 e $366 \mathrm{~nm})$ e nebulização com anisaldeído sulfúrico seguida de aquecimento (120 
$\left.{ }^{\circ} \mathrm{C}\right)$. Sílica gel (230-400 mesh ASTM, tamanho de partícula 0,040$0,063 \mu \mathrm{m}$, Merck) ou Sephadex LH-20 (Pharmacia) foram usadas em fracionamento por coluna cromatográfica. As separações por cromatografia líquida de alta eficiência (CLAE) foram realizadas em cromatógrafo analítico ternário Varian Pro Star modelo 240, com auto injetor Varian Pro Star modelo 410, detector UV-Diode-Array modelo 330, ou cromatógrafo analítico Shimadzu, bombas LC-10AD, com auto-injetor SIL 10A/ CBM 10A, detector Diode-Array modelo SPDM 10AVP e colunas analítica e preparativa "Luna" Phenomenex (250 $\mathrm{mm} \times 4,6 \mathrm{~mm}$ e $250 \mathrm{~mm}$ x $21 \mathrm{~mm}$, respectivamente).

\section{Material vegetal}

Folhas de Swartzia langsdorffii foram coletadas na Reserva da Juréia, Iguape - SP, em outubro de 2004. A identificação foi realizada pela Dra. I. Cordeiro (IBt - SMA, SP) e a excicata (Cordeiro 1913) foi depositada no herbário do Instituto de Botânica da Secretaria do Meio Ambiente, IBt - SMA São Paulo.

\section{Extração e isolamento dos constituintes químicos}

O material vegetal seco e moído ( $270 \mathrm{~g}$ ) foi extraído com EtOH a temperatura ambiente. Após concentração sob pressão reduzida, o extrato etanólico $(4,4 \mathrm{~g})$ foi submetido à partição com solventes orgânicos, fornecendo as frações obtidas com hexano (FH, 1,5 g), AcOEt (FA, 1,0 g) e $\mathrm{MeOH} / \mathrm{H}_{2} \mathrm{O}$ (FM, 1,5 g). A avaliação da atividade antifúngica do extrato bruto e das frações obtidas de partição revelou a presença de substâncias bioativas em FA e FM. O fracionamento de FA por cromatografia em coluna (sílica-gel, gradiente Hexano/AcOEt/ $\mathrm{MeOH}$ ) forneceu 12 frações (A1-A12). A análise por CCD (AcOEt/ $\mathrm{MeOH}$ 13:7) e CLAE (C-18, MeOH/H $\mathrm{H}_{2} \mathrm{O} 55: 45$, detecção em 235 nm) e o resultado da avaliação de atividade antifúngica orientaram a seleção de A6 e A7, que foram reunidas e purificadas por CLAE $\left(\mathrm{C}_{18}\right.$, $\mathrm{MeOH} / \mathrm{H}_{2} \mathrm{O} 55: 45$, detecção em $235 \mathrm{~nm}$, fluxo $12 \mathrm{~mL} / \mathrm{min}$ ), fornecendo a substância 2 (17 mg). O fracionamento de FM (1,5 g) por cromatografia em coluna (Sephadex LH-20, eluição com $\mathrm{MeOH}$ ) forneceu 49 subfrações, cuja análise por CCD (AcOEt/MeOH 9:11) orientou seu agrupamento em 9 frações (M1-M9). A análise das frações reunidas por CLAE (C-18, MeOH/ $\mathrm{H}_{2} \mathrm{O}$ 43:57, detecção em $235 \mathrm{~nm}$ ) e o resultado da avaliação de atividade antifúngica orientaram a seleção da fração M6 (55 mg), que foi purificada por CLAE $\left(\mathrm{C}_{18}, \mathrm{MeOH} / \mathrm{H}_{2} \mathrm{O}\right.$ 43:57, detecção em $235 \mathrm{~nm}$, fluxo $12 \mathrm{~mL} / \mathrm{min}$ ), fornecendo a substância $1(22 \mathrm{mg})$. O fracionamento de $\mathrm{FH}(1,5 \mathrm{~g})$ por cromatografia em coluna (sílica-gel, gradiente Hexano/AcOEt) forneceu 30 subfrações, que foram analisadas por CCD e agrupadas em 4 frações (H1-H4). A análise por CCD (Hexano/AcOEt 4:1) orientou a seleção de H3 e H4, que foram purificadas por CCD preparativa (Hexano/AcOEt 9:1, 3 eluições), fornecendo as substâncias 3 (17 mg) e 4 (8 mg).

\section{Atividade antifúngica}

Ensaio com fungos patogênicos humanos em microplacas

Os microrganismos usados na avaliação de atividade antifúngica foram Candida albicans ATCC 90028, Candida krusei ATCC 6258, Candida parapsilosis ATCC 22019 e Cryptococcus neoformans ATCC 90012, crescidos e mantidos em meio de ágar Sabouraud-dextrose por $24 \mathrm{~h}$ para as espécies de Candida e por $48 \mathrm{~h}$ para Cryptococcus neoformans, em temperatura ambiente. O método utilizado para avaliação da atividade foi microdiluição em caldo, descrito no documento M27-A2 do Clinical and Laboratory Standards Institute (CLSI), ${ }^{12}$ com modificações. O meio usado foi RPMI 1640 com Lglutamina em tampão a pH 7,0 com ácido morfolinopropanosulfônico (MOPS) 0,165 M, suplementado com glicose $2 \%$. Os ex- tratos, frações ou substâncias puras foram dissolvidos em DMSO em concentrações decrescentes a partir de 250 até $0,4 \mu \mathrm{g} / \mathrm{mL}$, e adicionados às microplacas de 96 cavidades contendo meio RPMI. As suspensões celulares contendo fungos foram preparadas em solução salina $0,85 \%$ e diluídas 1:100 em RPMI até concentração final de $1 \times 10^{4}$ a $5 \times 10^{4}$ unidades formadoras de colônias por $\mathrm{mL}(\mathrm{CFU} / \mathrm{mL})$. Cada suspensão foi inoculada nas microplacas previamente preparadas com as amostras teste, que foram incubadas com agitação a 37 ${ }^{\circ} \mathrm{C}$ por $24 \mathrm{~h}$ para Candida sp. e $48 \mathrm{~h}$ para Cryptococcus neoformans. Anfotericina B $(0,03$ a $16 \mu \mathrm{g} / \mathrm{mL})$ foi usada como droga controle. A concentração inibitória mínima (CIM) foi definida como a menor concentração de amostra que inibiu completamente o crescimento do fungo. Pelo método espectrofotométrico, a CIM foi definida como a menor concentração em que a densidade óptica foi reduzida a $90 \%$ daquela observada para a solução controle (sem amostra). Os resultados foram analisados visual e espectrofotometricamente. Amostras que apresentaram CIM menor que $75,0 \mu \mathrm{g} / \mathrm{mL}$ foram consideradas com boa atividade; CIM entre 75,0 e $150,0 \mu \mathrm{g} / \mathrm{mL}$ indicou atividade moderada; CIM entre 150,0 e $250,0 \mu \mathrm{g} / \mathrm{mL}$ indicou atividade fraca e, para CIM maior que $250,0 \mu \mathrm{g} / \mathrm{mL}$ a amostra foi considerada inativa.

\section{Bioautografia com fungos fitopatogênicos}

Os microrganismos usados na avaliação de atividade antifúngica foram Cladosporium cladosporioides e C. sphaerospermum, crescidos e mantidos em meio de ágar Sabouraud-dextrose. Cada amostra foi submetida à análise em CCD e, depois de seca, a placa cromatográfica foi nebulizada com suspensão dos esporos dos fungos Cladosporium cladosporioides ou C. sphaerospermum. As placas foram mantidas a $25{ }^{\circ} \mathrm{C}$ no escuro por $48-72 \mathrm{~h}$. Foi possível observar o crescimento dos fungos sobre as placas, exceto nos locais em que substâncias antifúngicas estavam presentes, os quais permaneceram brancos. As zonas de inibição foram medidas e comparadas com as substâncias padrão nistatina e miconazol..$^{13}$

\section{RESULTADOS E DISCUSSÃO}

$\mathrm{O}$ fracionamento cromatográfico do extrato $\mathrm{EtOH}$ das folhas de $S$. langsdorffii forneceu o 3-O- $\beta$-D-(6'-metil)-glicopiranosilolean-28-oato de $\beta$-glucopiranosila (1), ácido 3-O- $\beta$-glicopiranosil$(1 \rightarrow 2)-\beta$-D-glucopiranosil-oleanólico (2, ácido 3-soforosiloleanólico), ácido oleanólico (3) e lupeol (4).

A substância 1 foi obtida como sólido amorfo, com $[\alpha]_{D}{ }^{30}+31$ $(\mathrm{MeOH}, c 0,021)$ e seu espectro de massas mostrou um íon $[\mathrm{M}-\mathrm{H}]^{+}$ em $\mathrm{m} / z, 793$ (100), enquanto o espectro de massas de alta resolução mostrou sinal do íon $[\mathrm{M}]^{+}$em $\mathrm{m} / \mathrm{z}$ 794,4788, compatível com a fórmula molecular $\mathrm{C}_{43} \mathrm{H}_{70} \mathrm{O}_{13}$ (massa calculada 794,4816), consistente com a estrutura de uma saponina (Figura 1). Absorções na

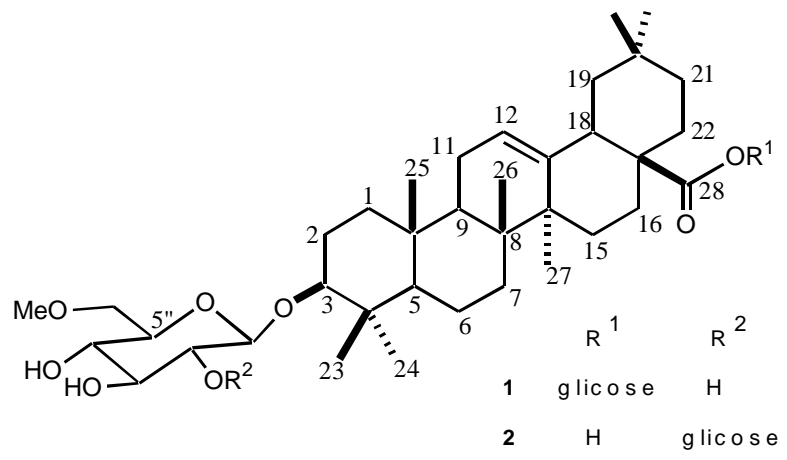

Figura 1. Estruturas das saponinas isoladas de Swartzia langsdorffii 
região de infra-vermelho em $1736 \mathrm{~cm}^{-1}$ indicaram a presença de um grupo carbonílico de éster. A análise do espectro de RMN de ${ }^{1} \mathrm{H}$ da substância 1 mostrou sinais para sete metilas alifáticas $\left(\delta_{H}\right.$ $0,8$ a 1,1$)$, sinais para hidrogênios hidroximetínicos $\left(\delta_{\mathrm{H}} 3,0\right.$ e 4,5), além de outros sinais entre $\delta_{\mathrm{H}} 1,0$ e 2,5 sugerindo a estrutura de um triterpeno glicosilado (saponina) (Tabela 1).

$\mathrm{O}$ espectro de RMN de ${ }^{13} \mathrm{C}$ mostrou sinais em $\delta_{\mathrm{C}} 121,7$ e 143,4 sugerindo o esqueleto oleanano para a substância 1; um sinal em $\delta_{\mathrm{C}}$ 175,3, compatível com uma carboxila e sinais em $\delta_{C}$ 94,1 e 105,3, que mostraram correlação com os dubletos em $\delta_{\mathrm{H}} 5,23(J=7,5 \mathrm{~Hz})$ e $\delta_{\mathrm{H}}$ $4,13(J=7,5 \mathrm{~Hz})$ no mapa de correlações HMQC, que permitiu suge-

Tabela 1. Dados de RMN de ${ }^{1} \mathrm{H}(500 \mathrm{MHz})$ e ${ }^{13} \mathrm{C}(125 \mathrm{MHz}) \mathrm{da}$ substância 1 em DMSO-d ${ }_{6} ; \delta$ (ppm)

\begin{tabular}{|c|c|c|c|}
\hline Posição & $\delta_{\mathrm{C}}$, mult. ${ }^{\mathrm{a}}$ & $\delta_{H}^{b}$ & HMBC \\
\hline 1 & $38,2\left(\mathrm{CH}_{2}\right)$ & $0,74(\mathrm{~m}) ; 1,32(\mathrm{~m})$ & H-23; H-25 \\
\hline 2 & $27,2\left(\mathrm{CH}_{2}\right)$ & $1,48(\mathrm{~m}) ; 1,85(\mathrm{~m})$ & $\mathrm{H}-25$ \\
\hline 3 & $87,8(\mathrm{CH})$ & 3,02 (ddl) & H-1”; H-23; H-24 \\
\hline 4 & $38,7(\mathrm{C})$ & - & $\mathrm{H}-24$ \\
\hline 5 & $55,0(\mathrm{CH})$ & $0,71(\mathrm{~m})$ & H-23; H-24; H-25 \\
\hline 6 & $17,8\left(\mathrm{CH}_{2}\right)$ & $1,23(\mathrm{~m}) ; 1,78(\mathrm{~m})$ & \\
\hline 7 & $31,8\left(\mathrm{CH}_{2}\right)$ & $1,85(\mathrm{~m})$ & H-26 \\
\hline 8 & $39,0(\mathrm{C})$ & - & $\mathrm{H}-27$ \\
\hline 9 & 47,1 (CH) & $1,48(\mathrm{~m})$ & H-26 \\
\hline 10 & $36,3(\mathrm{C})$ & - & $\mathrm{H}-25$ \\
\hline 11 & $22,4\left(\mathrm{CH}_{2}\right)$ & $1,78(\mathrm{dl}) ; 1,95(\mathrm{~m})$ & \\
\hline 12 & $121,7(\mathrm{CH})$ & $5,16(\mathrm{tl})$ & \\
\hline 13 & $143,4(\mathrm{C})$ & - & $\mathrm{H}-27$ \\
\hline 14 & $41,3(\mathrm{C})$ & - & H-26; H-27 \\
\hline 15 & $27,2\left(\mathrm{CH}_{2}\right)$ & $1,23(\mathrm{~m}) ; 1,95(\mathrm{~m})$ & $\mathrm{H}-27$ \\
\hline 16 & $23,0\left(\mathrm{CH}_{2}\right)$ & $0,98(\mathrm{~m}) ; 1,85(\mathrm{~m})$ & \\
\hline 17 & $45,9(\mathrm{C})$ & - & \\
\hline 18 & $40,7(\mathrm{CH})$ & 2,73 (ddl) & \\
\hline 19 & $45,6\left(\mathrm{CH}_{2}\right)$ & $1,08(\mathrm{~m}) ; 1,60(\mathrm{~m})$ & $\mathrm{H}-30$ \\
\hline 20 & $30,3(\mathrm{C})$ & - & H-29; H-30 \\
\hline 21 & $33,1\left(\mathrm{CH}_{2}\right)$ & $0,98(\mathrm{~m})$ & \\
\hline 22 & $32,1\left(\mathrm{CH}_{2}\right)$ & $0,74(\mathrm{~m})$ & \\
\hline 23 & $27,6\left(\mathrm{CH}_{3}\right)$ & $0,96(\mathrm{~s})$ & $\mathrm{H}-24$ \\
\hline 24 & $16,7\left(\mathrm{CH}_{3}\right)$ & $0,68(s)$ & $\mathrm{H}-23$ \\
\hline 25 & $15,2\left(\mathrm{CH}_{3}\right)$ & $0,86(\mathrm{~s})$ & \\
\hline 26 & $16,5\left(\mathrm{CH}_{3}\right)$ & $0,75(\mathrm{~s})$ & \\
\hline 27 & $25,5\left(\mathrm{CH}_{3}\right)$ & $1,08(\mathrm{~s})$ & \\
\hline 28 & $175,3(\mathrm{C})$ & - & H-1' \\
\hline 29 & $32,8\left(\mathrm{CH}_{3}\right)$ & $0,87(\mathrm{~s})$ & \\
\hline 30 & $23,4\left(\mathrm{CH}_{3}\right)$ & $0,86(\mathrm{~s})$ & H-29 \\
\hline $1{ }^{\prime}$ & $94,1(\mathrm{CH})$ & $5,23(\mathrm{~d}, 7,5)$ & \\
\hline $2^{\prime}$ & $72,4(\mathrm{CH})$ & $3,1(\mathrm{~m})$ & \\
\hline $3^{\prime}$ & $76,8(\mathrm{CH})$ & $3,22(\mathrm{~m})$ & \\
\hline $4^{\prime}$ & 69,6 (CH) & $3,12(\mathrm{~m})$ & \\
\hline $5^{\prime}$ & $77,7(\mathrm{CH})$ & $3,14(\mathrm{~m})$ & \\
\hline $6{ }^{\prime}$ & $60,7\left(\mathrm{CH}_{2}\right)$ & $3,42(\mathrm{~m}) ; 3,62(\mathrm{~m})$ & \\
\hline $1 "$ & $105,3(\mathrm{CH})$ & $4,13(\mathrm{~d}, 7,5)$ & \\
\hline $2 "$ & 73,9 (CH) & $2,95(\mathrm{tl})$ & \\
\hline $3 "$ & $76,7(\mathrm{CH})$ & $3,14(\mathrm{~m})$ & \\
\hline $4 "$ & $72,1(\mathrm{CH})$ & $3,11(\mathrm{~m})$ & \\
\hline $5 "$ & $74,1(\mathrm{CH})$ & $3,21(\mathrm{tl})$ & \\
\hline $6 "$ & $67,0\left(\mathrm{CH}_{2}\right)$ & $3,40(\mathrm{~m}) ; 3,62(\mathrm{~m})$ & \\
\hline $\mathrm{OMe}$ & $53,4\left(\mathrm{CH}_{3}\right)$ & $3,1(\mathrm{~s})$ & H-6" \\
\hline
\end{tabular}

a multiplicidades obtidas pelos experimentos DEPT $135^{\circ}$ e $90^{\circ}$; ${ }^{\mathrm{b}}$ multiplicidades e constantes de acoplamento $(\mathrm{J}$, em Hz), entre parênteses. rir a presença de duas unidades glicosídicas em 1. ${ }^{14,15}$ Observou-se ainda neste mapa de contorno um sinal em $\delta_{\mathrm{CH} 3} 53,4$ correlacionado ao singleto em $\delta_{\mathrm{H}} 3,1$, correspondente ao grupo metoxila, além de sinais para carbonos metínicos em $\delta_{\mathrm{CH}} 40,7$ e 47,1 , correlacionados ao duplo dubleto largo em $\delta_{\mathrm{H}} 2,73$ e ao multipleto em $\delta_{\mathrm{H}} 1,48$, que permitiram sua atribuição a $\mathrm{H}-18$ e H-9, respectivamente. O experimento HMBC mostrou correlações dos dubletos em $\delta_{\mathrm{H}} 5,23$ e 4,13, atribuídos aos hidrogênios anoméricos, com os sinais em $\delta_{\mathrm{C}} 175,3$ (C) e 87,8 $(\mathrm{CH})$ que foram atribuídos à carboxila do $\mathrm{C}-28$ e ao C-3, respectivamente, evidenciando as posições de substituição das duas unidades glicosídicas na aglicona triterpênica (Figura 2). Adicionalmente, este experimento mostrou a correlação entre o sinal em $\delta_{\mathrm{CH} 2} 67,0$ (C-6"), e o singleto em $\delta_{\mathrm{H}} 3,1$, permitindo a proposta de metilação na hidroxila em C-6". A análise detalhada deste mapa mostrou correlações entre os hidrogênios metílicos e carbonos a 2 ou 3 ligações, o que, em conjunto com a comparação dos seus dados espectrométricos com a literatura, ${ }^{2,15}$ permitiu confirmar a estrutura proposta para a substância $\mathbf{1}$, o ácido 3-O- $\beta$-D-(6'-metil)glicopiranosil-28- $O$ - $\beta$-D-glucopiranosiloleanólico, inédita na literatura.

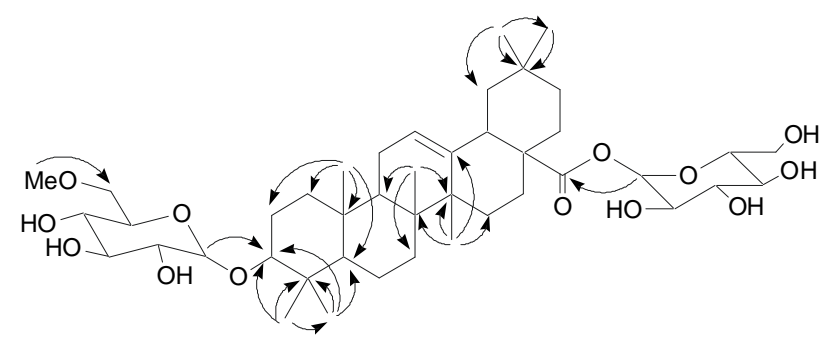

Figura 2. Correlações observadas no espectro HMBC da substância 1

A substância 2 foi obtida como sólido amorfo e seu espectro de massas mostrou um pico em $\mathrm{m} / z .779$ (100\%) que pode ser atribuído ao íon $[\mathrm{M}-\mathrm{H}]^{+}$. Este valor é compatível com a fórmula molecular $\mathrm{C}_{42} \mathrm{H}_{68} \mathrm{O}_{13}$, que está de acordo com a estrutura de uma saponina triterpênica com duas unidades de glicose. Os dados de RMN de ${ }^{1} \mathrm{H}$ e de ${ }^{13} \mathrm{C}$ foram semelhantes aos da saponina $\mathbf{1}$, evidenciando uma aglicona com esqueleto oleanano, através dos sinais em $\delta_{\mathrm{C}}$ 123,6 e 145,2, atribuídos a C-12 e C-13 e a presença de duas unidades glicosídicas, em função dos sinais para dois hidrogênios $\left(\delta_{\mathrm{H}}\right.$ 4,37 e 4,56) e dois carbonos anoméricos $\left(\delta_{\mathrm{C}} 106,3\right.$ e 103,1). O experimento HMBC indicou correlações entre C-3 e H-1' e entre C-2' e H-1", que evidenciaram a posição da ligação da aglicona com uma unidade glicosídica, bem como da ligação interglicosídica, e permitiram identificar a substância 2 com o ácido 3-O- $\beta$ glicopiranosil-( $1 \rightarrow 2)-\beta$-D-glucopiranosil-oleanólico, anteriormente isolado de S. schomburgkii. ${ }^{2,15}$

As substâncias $\mathbf{3}$ e $\mathbf{4}$ foram obtidas como sólidos amorfos e seus dados de EM obtidos por ionização por eletrospray e de RMN uni e bidimensionais, bem como comparação com dados da literatura, ${ }^{14}$ permitiram identificá-las como os triterpenos pentacíclicos ácido oleanólico (3) e lupeol (4), amplamente distribuídos no reino vegetal.

A seleção do extrato de $S$. langsdorffii para estudo fitoquímico visando o isolamento de seus constituintes potencialmente antifúngicos baseou-se no resultado positivo (CIM 125,0 $\mu \mathrm{g} / \mathrm{mL}$ ) obtido frente às linhagens de fungos patogênicos humanos Candida albicans, Candida krusei, Candida parapsilosis e Cryptococcus neoformans, indicando atividade antifúngica moderada. A avaliação das fases de partição, FA e FM (CIM 125,0 $\mu \mathrm{g} / \mathrm{mL})$, confirmou a bioatividade observada inicialmente e orientou a escolha das frações promissoras para purificação das substâncias bioativas. As substâncias purificadas 1-4 também foram avaliadas por suas pro- 
priedades antifúngicas. As saponinas $\mathbf{1}$ e $\mathbf{2}$, obtidas de FM e FA, respectivamente, mostraram-se moderadamente ativas (CIM 100,0 $\mu \mathrm{g} / \mathrm{mL}$ ), enquanto a substância $\mathbf{3}$ se mostrou fracamente ativa (CIM $200,0 \mu \mathrm{g} / \mathrm{mL}$ ) e $\mathbf{4}$ foi inativa frente aos fungos patogênicos usados neste estudo.

As substâncias 1-4 foram também avaliadas por bioautografia frente aos fungos fitopatogênicos Cladosporium cladosporioides e C. sphaerospermum. As substâncias 1-3 mostraram-se moderadamente ativas, com halos de inibição de 15,12 e $11 \mathrm{~mm}$, enquanto 4 mostrou-se inativa, confirmando a tendência observada anteriormente da atividade antifúngica do ácido oleanólico e seus derivados. ${ }^{16}$

\section{CONCLUSÃO}

O estudo da espécie $S$. langsdorffii mostrou a presença de saponinas nas subfrações provenientes das fases de partição em AcOEt e aquosa. Verificou-se ainda a presença de triterpenos pentacíclicos na fase hexânica, sendo isolados e identificados o lupeol e o ácido oleanólico. A avaliação da atividade antifúngica do extrato bruto das folhas de S. langsdorffii e de suas fases de partição orientou a busca das substâncias bioativas, que resultou no isolamento das saponinas antifúngicas a partir das frações mais polares. Esses resultados corroboram a importância do estudo fitoquímico guiado por bioensaios como estratégia efetiva para atividades de bioprospecção.

\section{AGRADECIMENTOS}

Este estudo foi realizado com apoio financeiro da FAPESP, programa BIOTA/BIOprospecTA, através dos projetos 03/02176-7 e 04/07932-7; CNPq e CAPES, através de verbas de auxílio à pesquisa e bolsas de estudo. Os autores agradecem à Dra. I. Cordeiro (IBt - SMA, São Paulo) pela identificação do material botânico.

\section{REFERÊNCIAS}

1. Veiga, V. F.; Pinto, A. C.; Quim. Nova 2002, 25, 273.

2. Abedl-Kader, M. S.; Bahler, B. D.; Malone, S.; Werkhoven, M. C. M.; Wisse, J. H.; Nedmann, K. M.; Bursuker, I.; Kingston, D. G. I.; J. Nat. Prod. 2000, 63, 1461.

3. Tozzi, A. M. G. A.; Rev. Bras. Bot. 1999, 22, 2 .

4. Magalhães, A. F.; Tozzi, A. M.; Santos, C. C.; Magalhães, E. G.; J. Nat. Prod. 2005, 68, 1290; Osawa, K.; Yasuda, H.; Maruyama, T.; Morita, H.; Takeya, K.; Itokawa, H.; Chem. Pharm. Bull. 1992, 40, 2970.

5. Braz Filho, R.; Moraes, M. P. L.; Gottlieb, O. R.; Phytochemistry 1980, 19, 2003; Donnelly, D. M.; Fitzgerald, M. A.; Phytochemistry 1971, 10, 3147; Formiga, M. D.; Gottlieb, O. R.; Mendes, P. H.; Koketsu, M.; Almeida, M. E. L.; Pereira, M. O. S.; Magalhães, M. T.; Phytochemistry 1975, 14, 828. Schaller, F.; Wolfender, J. L.; Hostettmann, K.; Mavi, S.; Helv. Chem. Acta 2001, 84, 222.

6. Hostettmann, K.; Wolfender, J. L.; Pestic. Sci. 1997, 51, 471.

7. Dubois, J. L.; Sneden, A. T.; J. Nat. Prod. 1995, 58, 629; Dubois, J. L.; Sneden, A. T.; J. Nat. Prod. 1996, 59, 902.

8. Ficker, C. E.; Arnason, J. T.; Vindas, P. S.; Alvarez, L. P.; Akpagana, K.; Gbeassor, M.; De Souza, C.; Smith, M.; Mycoses 2003, 16, 26.

9. Trabulsi, L. R.; Alterthum, F.; Gompertz, O. F.; Candeias, J. A. N.; Microbiologia, $3^{\text {a }}$ ed, Atheneu: São Paulo, 1999.

10. Schmourlo, G.; Mendonça Filho, R. R.; Alviano, C. S.; Costa. S.; J. Ethnopharmacol. 2005, 96, 563.

11. Duarte, M. C. T.; Figueira, G. M.; Sartoratto, A.; Rehder, V. L. G.; Delarmelina, C.; J. Ethnopharmacol. 2005, 97, 305; Pinto, A. C.; Silva, D. H. S.; Lopes, N. P.; Epifanio, R. A.; Bolzani, V. S.; Quim. Nova 2002, 25,45 .

12. Reference method for broth dilution antifungal susceptibility testing of yeasts. Approved standard-NCCLS document M27-A2; National Committee for Clinical Laboratory Standards, 2002, $2^{\text {nd }}$ ed., Clinical and Laboratory Standards Institute: Villanova, $\mathrm{Pa}$.

13. Homans, A. L.; Fuchs, A.; J. Chromatogr. 1970, 51, 325.

14. Breitmaier, E.; Voelter, W.; Carbon-13 NMR Spectroscopy, $3^{\text {rd }}$ ed.; VCH Vedagsgesellchaft mbH: Weinheim, 1989, p. 379-394; Mahato, S. B.; Kundu, A. P.; Phytochemistry 1994, 37, 1517.

15. Magalhães, A. F.; Tozzi, A. M. G. A.; Santos, C. C.; Serrano, D. R.; ZanottiMagalhães, E. M.; Magalhães, E. G.; Magalhães, L. A.; Mem. Inst. Oswaldo Cruz 2003, 98, 5; Nagao, T.; Tanaka, R.; Iwase, Y.; Hanazono, H.; Okabe, H.; Chem. Pharm. Bull. 1991, 39, 599; Orsini, F.; Pelizzoni, F.; Ricca, G.; Verotta, L.; Phytochemistry 1987, 26, 1101.

16. Johann, S.; Soldi, C.; Lyon, J. P.; Pizzolatti, M. G.; Resende, M. A.; Lett. Appl. Microbiol. 2007, 45, 148. 